\section{Handwriting as a marker for PD progression: a shift in paradigm}
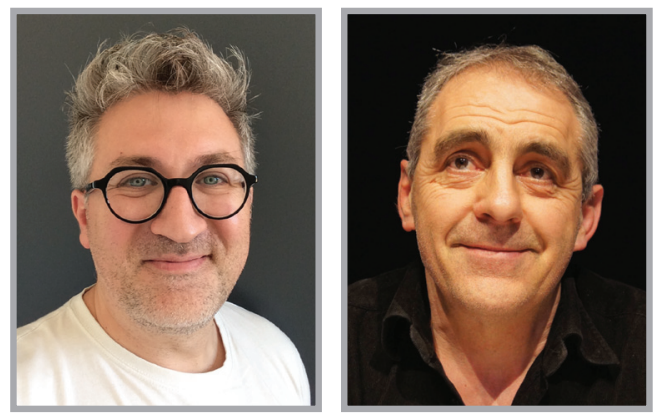

“' It has been reported that short and routine writing tasks based on kinematics analyses could help to distinguish PD patients from controls."

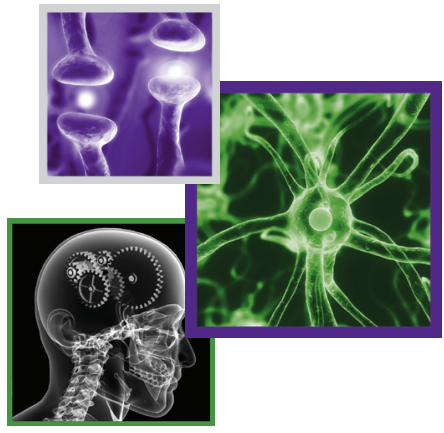

Serge Pinto ${ }^{*, 1} \&$ Jean-Luc Velay**,2

Parkinson's disease (PD) is the second most common neurodegenerative disease after Alzheimer's disease, its prevalence reaching $1 \%$ of individuals over 60 years old [1]. In recent decades, understanding the neurodegenerative processes of PD has been the subject of extensive research, thus contributing to improvements in PD diagnosis, treatment and management. Against this backdrop, identifying sensitive biomarkers in PD (e.g., genetic, clinical, biochemical, physiological) to track disease occurrence and progression is currently of great interest in the research community (for a comprehensive review, see [2]). This kind of research represents the core of collaborative projects that gather together large teams with common objectives (e.g., the longitudinal program for biomarker development in Parkinson's disease [3] and the Parkinson Progression Marker Initiative [4]). In the biomarker classification provided by Sharma et al., one can find agraphia, impaired handwriting and micrographia among the presymptomatic neurobehavioral biomarkers [2].

Micrographia in Parkinson's disease: too restrictive a definition

Although handwriting impairment is a frequent initial sign that prompts patients to seek the advice of a physician, it is classically not included in the diagnosis of PD. Consequently, as part of the identification of new and objective biomarkers to help diagnose PD at its earliest stage and manage disease progression, handwriting analysis has been proposed [5-7]. Until recently, there was a consensus regarding the existence of a specific behavioral feature in PD handwriting, so-called micrographia. This term refers to 'an obvious reduction in size of the lettering of the writer in comparison with his calligraphy before the development of the organic lesion effecting the change' $[8]$. It has been used generically to define all aspects of handwriting in PD, although it is only one of the prominent features of handwriting impairment.

\section{KEYWORDS}

- biomarker • diagnosis • dysgraphia

- handwriting • micrographia

- Parkinson's disease

"We concluded that although studies focusing on writing size did contribute to a meaningful description of PD micrographia, handwriting impairment in PD cannot be restricted only to size reduction anymore." 
Micrographia has further been divided into two types: consistent micrographia, presenting as a global reduction in writing size, and progressive micrographia, which results from an inability to sustain normal sized letters for more than a few characters $[9,10]$. The occurrence of micrographia varies between studies: 10-15 [11], 29 [12], 44 [9] or even $63.2 \%$ [13]. This large range mainly results from the number of patients studied, the heterogeneity of symptoms and the nature of the analyses (quantitative vs self-questionnaires). In fact, according to the published studies, it should be noted that less than $50 \%$ of patients studied presented micrographia [14].

\section{From micrographia to dysgraphia in Parkinson's disease}

Micrographia is easy to detect with conventional (paper and pencil) tools, and the first studies that investigated PD patients' handwriting were based on the written traces, that is, the static product of handwriting movements. Following the development of graphic tablets, several 'hidden' variables of handwriting movement that are not visible in the written trace have been made available for assessment, allowing for a new kind of handwriting analysis that characterizes the handwriting process, that is, the movement that generates the trace. Indeed, based on studies carried out during the last decade, we proposed that four main variables are of crucial interest for analysis of handwriting impairment: size, duration, speed and fluency [15]. More recently, from an exhaustive identification of the studies investigating the impact of PD on handwriting, we questioned the meaning of micrographia in PD and the relevance of continuing to use this term in light of the findings raised by graphics tablet evaluations [14].

Defining specific handwriting dysfunctions in $\mathrm{PD}$, and the parameters that could distinguish PD patients from controls, has been the main objective of multiple studies, though nearly half of these studies (43\%) failed to highlight any significant difference between PD patients and age-matched controls as regards to the writing size. Similarly, for writing duration, $56 \%$ failed to demonstrate any significant difference between patients and age-matched controls [14]. However, the comparisons of kinematic variables (velocity, fluency) between control subjects and PD patients, as well as between treatment states (with and without medication), were much more distinguishable. We concluded that although studies focusing on writing size did contribute to a meaningful description of PD micrographia, handwriting impairment in PD cannot be restricted only to size reduction anymore. Therefore, we proposed to adopt the term PD 'dysgraphia' in order to encompass all deficits affecting PD handwriting. "This position is not only semantic, it has two consequences: one theoretical and one practical. First, it implies that motor impairments related to the disease (e.g., tremor, rigidity, bradykinesia, akinesia, freezing of the upper limb, etc.) may affect the handwriting kinematics without necessarily affecting its size. Second, it implies that dynamical and kinematical variables are more suited for the diagnosis and follow-up of $\mathrm{PD}$, or for evaluating the efficiency of a given treatment" [14].

\section{Dysgraphia as a potential presymptomatic marker of Parkinson's disease}

According to Gerlach et al., "an ideal biomarker to be useful to assess neurodegeneration in PD and to evaluate disease-modifying therapeutics (...) should be: linked to fundamental features of PD neuropathology and mechanisms underlying neurodegeneration in PD; correlated to disease progression assessed by clinical rating scales; able to monitor the actual disease status; preclinically validated; confirmed by at least two independent studies conducted by qualified investigators with the results published in peer-reviewed journals" [16]. The authors also stated that this ideal biomarker "should be inexpensive, noninvasive, simple to use and technically validated (e.g., reliable, sensitive to change)" [16].

If we consider dysgraphia in PD from this point of view, we reach the following conclusions: handwriting impairment was found to be positively correlated with an accurate diagnosis of PD $[17,18]$; correlations between handwriting variables and clinical evaluations in PD patients (motor part of the Unified Parkinson's Disease Rating Scale [19]) were found in several studies (e.g., $[9,12]$ ); changes in handwriting increase with PD progression [7]; there is still a need to confirm the preclinical status of handwriting impairment; many studies led by experts have specifically targeted an understanding of handwriting impairment in PD (for a review, see $[14,20]$ ). In addition, it has been reported that short and routine writing tasks based on kinematics analyses could help to distinguish PD patients from controls [7]. 
In line with this approach, we also suggested that computerized analysis of handwriting movements represents a simple, noninvasive and useful tool that can contribute to both PD diagnosis and follow-up for clinicians who deal with PD dysgraphia [14]. Taken together, these findings turn PD dysgraphia into a reliable physiological biomarker for the early detection of PD [7,14].

\section{Acknowledgements}

The authors would like to thank J Danna (Laboratoire de Neurosciences Cognitives - LNC - Aix-Marseille Université, CNRS, France) and A Letanneux (Laboratoire Parole et Langage - LPL - Aix-Marseille Université, CNRS, France) for their very straightforward work that helped to contribute to the proposal of such a 'shift in paradigm.' However, the authors would like to thank J Sneed German (Laboratoire Parole et Langage - LPL - AixMarseille Universite \& CNRS, France) for the English revision of the manuscript.

\section{Financial \& competing interests disclosure}

The authors have no relevant affliations or financial involvement with any organization or entity with a financial interest in or financial conflict with the subject matter or materials discussed in the manuscript. This includes employment, consultancies, honoraria, stock ownership or options, expert testimony, grants or patents received or pending, or royalties.

No writing assistance was utilized in the production of this manuscript.

\section{References}

1 Nussbaum RL, Ellis CE. Alzheimer's disease and Parkinson's disease. N. Engl. J. Med. 348, 1356-1364 (2003).

2 Sharma S, Moon CS, Khogali A et al. Biomarkers in Parkinson's disease (recent update). Neurochem. Int. 63(3), 201-229 (2013).

3 Ravina B, Tanner C, Dieuliis D et al. A longitudinal program for biomarker development in Parkinson's disease: a feasibility study. Mov. Disord. 24, 2081-2090 (2009).

4 Marek K, Jennings D, Lasch S et al. The Parkinson Progression Marker Initiative (PPMI). Prog. Neurobiol. 95, 629-635 (2011).

5 McLennan JE, Nakano K, Tyler HR, Schwab RS. Micrographia in Parkinson's disease. J. Neurol. Sci. 15(2), 141-152 (1972).

6 Phillips JG, Stelmach GE, Teasdale N. What can indices of handwriting quality tell us about Parkinsonian handwriting? Hum. Mov. Sci. 10(2-3), 301-314 (1991).

7 Rosenblum S, Samuel M, Zlotnik S, Erikh I, Schlesinger I. Handwriting as an objective tool for Parkinson's disease diagnosis. J. Neurol. 260(9), 2357-2361 (2013).

8 Wilson SK. The Croonian Lectures. On some disorder of motility and of muscle tone with special reference to the corpus. Lancet 2(1), $1-10(1925)$

9 Kim E-J, Lee BH, Park KC, Lee WY, Na DL. Micrographia on free writing versus copying tasks in idiopathic Parkinson's disease. Parkinsonism Relat. Disord. 11(1), 57-63 (2005).

10 Gangadhar G, Joseph D, Srinivasan AV et al. A computational model of Parkinsonian handwriting that highlights the role of the indirect pathway in the basal ganglia. Hum. Mov. Sci. 28(5), 602-618 (2009).

11 McLennan JE, Nakano K, Tyler HR, Schwab RS. Micrographia in Parkinson's disease. J. Neurol. Sci. 15(2), 141-152 (1972).

12 Wagle Shukla A, Ounpraseuth S, Okun MS, Gray V, Schwankhaus J, Metzer WS. Micrographia and related deficits in Parkinson's disease: a cross-sectional study. BJM Open 2(3) (2012).

13 Contreras-Vidal JL, Teulings HL, Stelmach GE. Micrographia in Parkinson's disease. Neuroreport 6(15), 2089-2092 (1995).

14 Letanneux A, Danna J, Velay JL, Viallet F, Pinto S. From micrographia to Parkinson's disease dysgraphia. Mov. Disord. 29, 1467-1475 (2014).

15 Danna J, Paz-Villagrán V, Velay J-L. Signal-to-Noise velocity peaks difference: a new method for evaluating the handwriting movement fluency in children with dysgraphia. Res. Dev. Disabil. 34(12), 4375-4384 (2013).

16 Gerlach M, Maetzler W, Broich Ket al. Biomarker candidates of neurodegeneration in Parkinson's disease for the evaluation of disease-modifying therapeutics. J. Neural Transm. 119, 39-52 (2012).

17 Duarte J, Claveria LE, Pedro-Cuesta D, Sempere AP, Coria F, Calne DB. Screening Parkinson's disease: a validated questionnaire of high specificity and sensitivity. Mov. Disord. 10(5), 643-649 (1995).

18 Mutch WJ, Smith WC, Scott RF. A screening and alerting questionnaire for Parkinsonism. Neuroepidemiology 10(3), 150-156 (1991).

19 Fahn S, Elton RL, Members of the UPDRS Development Committee. Unified Parkinson's Disease Rating Scale. In: Recent Developments in Parkinson's Disease. Fahn S, Marsden CD, Calne DB, Goldstein M (Eds.). Macmillan Healthcare Information, NJ, USA, 153-164 (1987).

20 Nackaerts E, Vervoort G, Heremans E, SmitsEngelsman BCM, Swinnen SP, Nieuwboer A. Relearning of writing skills in Parkinson's disease: a literature review on influential factors and optimal strategies. Neurosci. Biobehav. Rev. 37(3), 349-357 (2013). 